\title{
Small-Scale Molecular Motions Accomplish Glutamate Uptake in Human Glutamate Transporters
}

\author{
Hans P. Koch and H. Peter Larsson \\ Neurological Sciences Institute, Oregon Health and Science University, Beaverton, Oregon 97006
}

Glutamate transporters remove glutamate from the synaptic cleft to maintain efficient synaptic communication between neurons and to prevent glutamate concentrations from reaching neurotoxic levels. Glutamate transporters play an important role in ischemic neuronal death during stroke and have been implicated in epilepsy and amytropic lateral sclerosis. However, the molecular structure and the glutamate-uptake mechanism of these transporters are not well understood. The most recent models of glutamate transporters have three or five subunits, each with eight transmembrane domains, and one or two membrane-inserted loops. Here, using fluorescence resonance energy transfer (FRET) analysis, we have determined the relative position of the extracellular regions of these domains. Our results are consistent with a trimeric glutamate transporter with a large ( $>45 \AA$ ) extracellular vestibule. In contrast to other transport proteins, our FRET measurements indicate that there are no large-scale motions in glutamate transporters and that glutamate uptake is accompanied by relatively small motions around the glutamate-binding sites. The large extracellular vestibule and the small-scale conformational changes could contribute to the fast kinetics predicted for glutamate transporters. Furthermore, we show that, despite the multimeric nature of glutamate transporters, the subunits function independently.

Key words: EAAT3; FRET; uptake mechanism; independence; trimer; fluorescence

\section{Introduction}

Glutamate is the main excitatory neurotransmitter in the CNS. During neuronal activity, glutamate is released by exocytosis from presynaptic cells into the synapse, in which glutamate binds to and activates postsynaptic receptors. It is subsequently removed from the extracellular solution by glutamate transporters located in glial cells and neurons (Bergles et al., 1999; Danbolt, 2001). These transporters play an important role in shaping the time course of excitatory synaptic signals and are also believed to be involved in neurological disorders, such as amyotrophic lateral sclerosis and epilepsy (Tanaka et al., 1997; Watanabe et al., 1999; Danbolt, 2001). Glutamate transporters have also been implicated in contributing to the massive neuronal death that occurs during ischemia (Danbolt, 2001). These studies suggest that the removal of extracellular glutamate by glutamate transporters is essential for maintaining efficient synaptic communication between neurons and for preventing extracellular glutamate concentrations from reaching toxic levels. However, the molecular structure and the mechanism for glutamate uptake by these transporters are not well understood.

Five different members of glutamate transporters have been cloned (Kanai and Hediger, 1992; Pines et al., 1992; Storck et al., 1992; Arriza et al., 1994, 1997; Kanai et al., 1994; Fairman et al., 1995; Lin et al., 1998): excitatory amino acid transporters 1-5

Received 0ct. 5, 2004; revised Jan. 4, 2005; accepted Jan. 4, 2005.

This work was supported by a grant from the Medical Research Foundation (Oregon) and by National Institutes of Health Grants NS043259, NS051169, and RR016858. We thank J. Adelman, R. Duvoisin, C. Jahr, and A. Tzingounis for comments on this manuscript and $\mathrm{S}$. Oster for editing.

Correspondence should be addressed to H. Peter Larsson, Neurological Sciences Institute, Oregon Health and Science University, 505 NW 185th Avenue, Beaverton, 0R 97006. E-mail: larssonp@ohsu.edu.

D01:10.1523/JNEUROSCI.4138-04.2005

Copyright $\odot 2005$ Society for Neuroscience $\quad$ 0270-6474/05/251730-07\$15.00/0
(EAAT1-EAAT5). EAAT1 and EAAT2 are expressed in glial cells, whereas EAAT3 and EAAT4 are expressed in neurons (Rothstein et al., 1994; Chaudhry et al., 1995). EAAT5 is a retina-specific glutamate transporter.

Transport of one glutamate molecule is coupled to the cotransport of three sodium ions and one proton and to the countertransport of one potassium ion (Zerangue and Kavanaugh, 1996; Levy et al., 1998). The exact molecular mechanism for this coupled transport of substrates is not well understood. To gain more structural information about glutamate transporters and to test the nature of conformational changes that occur during glutamate uptake, we determined the relative positions of different domains in the human neuronal glutamate transporter EAAT3 under conditions that favor different conformations. To determine the relative positions of the different transmembrane domains (TMs) and membrane-inserted loops, we used fluorescence resonance energy transfer (FRET) analysis on EAAT3 transporters expressed in Xenopus oocytes. FRET is a phenomenon by which a donor fluorophore, in its excited state, transfers its excitation energy to a nearby acceptor fluorophore. The efficiency of FRET tapers off by the sixth power of the distance separating the donor and acceptor fluorophore, allowing an estimation of the relative distance between pairs of acceptor and donor fluorophores (Selvin, 1995).

Our results are consistent with a trimeric glutamate transporter with a large $(>45 \AA)$ extracellular vestibule. In contrast to other transport proteins (Kuhlbrandt et al., 2002; Toyoshima and Nomura, 2002; Abramson et al., 2003; Huang et al., 2003; Toyoshima and Inesi, 2004; Toyoshima and Mizutani, 2004), our FRET measurements indicate that there are no large-scale motions in glutamate transporters and that glutamate uptake is accompanied by relatively small motions around the glutamate- 
Table 1. FRET efficiencies and distance estimates

\begin{tabular}{|c|c|c|c|c|c|c|c|}
\hline Residue & $\tau_{1}(\mu \mathrm{M} \times \min )$ & $r_{\mathrm{d}}$ & $r_{\mathrm{a}}$ & $E$ & $R(\AA)$ & $L(\AA)$ & $\Delta R(\AA)$ \\
\hline S45C & $\begin{array}{l}3224 \pm 386 \\
\text { (3) }\end{array}$ & $\begin{array}{l}0.088 \pm 0.009 \\
(3)\end{array}$ & $\begin{array}{l}0.097 \pm 0.006 \\
(3)\end{array}$ & $\begin{array}{l}0.151 \pm 00.009 \\
(12)\end{array}$ & $74.8 \pm 4.6$ & $43.2 \pm 2.7$ & $\begin{array}{l}-0.9 \pm 1.4 \\
(5)\end{array}$ \\
\hline A53C & $\begin{array}{l}6973 \pm 830 \\
\text { (3) }\end{array}$ & $\begin{array}{l}0.080 \pm 0.009 \\
(3)\end{array}$ & $\begin{array}{l}0.209 \pm 0.004 \\
(3)\end{array}$ & $\begin{array}{l}0.190 \pm 00.011 \\
(14)\end{array}$ & $71.5 \pm 4.1$ & $41.3 \pm 2.4$ & $\begin{array}{l}-4.5 \pm 3.4^{*} \\
(5)\end{array}$ \\
\hline M60C & $\begin{array}{l}1150 \pm 57 \\
(2)\end{array}$ & $\begin{array}{l}0.094 \pm 0.009 \\
(3)\end{array}$ & $\begin{array}{l}0.135 \pm 0.009 \\
(3)\end{array}$ & $\begin{array}{l}0.590 \pm 00.034 \\
(14)\end{array}$ & $52.8 \pm 3.1$ & $30.5 \pm 1.8$ & ND \\
\hline T121C & $\begin{array}{l}370 \pm 20 \\
(2)\end{array}$ & $\begin{array}{l}0.065 \pm 0.004 \\
(3)\end{array}$ & $\begin{array}{l}0.162 \pm 0.010 \\
(3)\end{array}$ & $\begin{array}{l}0.189 \pm 00.010 \\
(15)\end{array}$ & $71.5 \pm 3.7$ & $41.3 \pm 2.1$ & $\begin{array}{l}-0.7 \pm 1.2 \\
(4)\end{array}$ \\
\hline S207C & $\begin{array}{l}393 \pm 39 \\
(2)\end{array}$ & $\begin{array}{l}0.047 \pm 0.004 \\
(3)\end{array}$ & $\begin{array}{l}0.158 \pm 0.012 \\
(3)\end{array}$ & $\begin{array}{l}0.319 \pm 00.023 \\
(15)\end{array}$ & $63.7 \pm 4.5$ & $36.8 \pm 2.6$ & $\begin{array}{l}2.0 \pm 1.1^{*} \\
(5)\end{array}$ \\
\hline V273C & $\begin{array}{l}6661 \pm 771 \\
(3)\end{array}$ & $\begin{array}{l}0.105 \pm 0.006 \\
\text { (3) }\end{array}$ & $\begin{array}{l}0.141 \pm 0.007 \\
(3)\end{array}$ & $\begin{array}{l}0.276 \pm 00.012 \\
(12)\end{array}$ & $65.9 \pm 3.0$ & $38.0 \pm 1.7$ & $\begin{array}{l}-1.2 \pm 00.7^{*} \\
(5)\end{array}$ \\
\hline $\mathrm{G} 283 \mathrm{C}$ & $\begin{array}{l}4685 \pm 412 \\
\text { (3) }\end{array}$ & $\begin{array}{l}0.117 \pm 0.010 \\
(3)\end{array}$ & $\begin{array}{l}0.097 \pm 0.007 \\
(3)\end{array}$ & $\begin{array}{l}0.267 \pm 00.016 \\
(19)\end{array}$ & $66.4 \pm 4.1$ & $38.3 \pm 2.4$ & $\begin{array}{l}-0.7 \pm 00.8 \\
(5)\end{array}$ \\
\hline S334C & $\begin{array}{l}1116 \pm 62 \\
(2)\end{array}$ & $\begin{array}{l}0.097 \pm 0.006 \\
(3)\end{array}$ & $\begin{array}{l}0.157 \pm 0.012 \\
\text { (3) }\end{array}$ & $\begin{array}{l}0.694 \pm 0.028 \\
(9)\end{array}$ & $49.0 \pm 2.0$ & $28.3 \pm 1.2$ & ND \\
\hline L387C & $\begin{array}{l}1892 \pm 211 \\
(3)\end{array}$ & $\begin{array}{l}0.065 \pm 0.009 \\
\text { (3) }\end{array}$ & $\begin{array}{l}0.146 \pm 0.010 \\
(3)\end{array}$ & $\begin{array}{l}0.433 \pm 00.026 \\
(12)\end{array}$ & $58.7 \pm 3.5$ & $33.9 \pm 2.0$ & $\begin{array}{l}-3.4 \pm 1.6^{* *} \\
(6)\end{array}$ \\
\hline Q413C & $\begin{array}{l}712 \pm 89 \\
(2)\end{array}$ & $\begin{array}{l}0.068 \pm 0.003 \\
(3)\end{array}$ & $\begin{array}{l}0.135 \pm 0.008 \\
(3)\end{array}$ & $\begin{array}{l}0.580 \pm 0.023 \\
(9)\end{array}$ & $53.2 \pm 2.1$ & $30.7 \pm 1.2$ & ND \\
\hline I421C & $\begin{array}{l}1778 \pm 122 \\
(2)\end{array}$ & $\begin{array}{l}0.049 \pm 0.003 \\
(3)\end{array}$ & $\begin{array}{l}0.102 \pm 0.005 \\
(3)\end{array}$ & $\begin{array}{l}0.548 \pm 00.037 \\
(13)\end{array}$ & $54.4 \pm 3.6$ & $31.4 \pm 2.1$ & ND \\
\hline A430C & $\begin{array}{l}1043 \pm 134 \\
\text { (4) }\end{array}$ & $\begin{array}{l}0.101 \pm 0.005 \\
\text { (3) }\end{array}$ & $\begin{array}{l}0.103 \pm 0.010 \\
(3)\end{array}$ & $\begin{array}{l}0.346 \pm 00.015 \\
(18)\end{array}$ & $62.4 \pm 2.7$ & $36.0 \pm 1.6$ & $\begin{array}{l}-0.8 \pm 00.5 \\
(4)\end{array}$ \\
\hline T434C & $\begin{array}{l}725 \pm 44 \\
(2)\end{array}$ & $\begin{array}{l}0.095 \pm 0.007 \\
(3)\end{array}$ & $\begin{array}{l}0.105 \pm 0.012 \\
\text { (3) }\end{array}$ & $\begin{array}{l}0.475 \pm 00.028 \\
(11)\end{array}$ & $57.1 \pm 3.4$ & $33.0 \pm 1.9$ & $\begin{array}{l}-1.2 \pm 1.3 \\
(5)\end{array}$ \\
\hline A430C plus V273C & & & & $\begin{array}{l}0.844 \pm 00.030 \\
(10)\end{array}$ & $33.7 \pm 0.7$ & & $\begin{array}{l}-1.0 \pm 1.5 \\
(5)\end{array}$ \\
\hline A430C plus G283C & & & & $\begin{array}{l}0.676 \pm 00.019 \\
(12)\end{array}$ & $39.0 \pm 0.2$ & & $\begin{array}{l}0.4 \pm 00.9 \\
(5)\end{array}$ \\
\hline G283C plus M60C & & & & $\begin{array}{l}0.615 \pm 00.028 \\
(11)\end{array}$ & $42.4 \pm 1.8$ & & $\begin{array}{l}-0.2 \pm 00.7 \\
(5)\end{array}$ \\
\hline A53C plus L387C & & & & $\begin{array}{l}0.440 \pm 00.019 \\
(12)\end{array}$ & $48.5 \pm 0.6$ & & $\begin{array}{l}-0.6 \pm 1.1 \\
(5)\end{array}$ \\
\hline S45C plus S207C & & & & $\begin{array}{l}0.516 \pm 00.012 \\
(9)\end{array}$ & $44.2 \pm 1.4$ & & $\begin{array}{l}0.5 \pm 00.9 \\
(5)\end{array}$ \\
\hline I421C plus M60C & & & & $\begin{array}{l}0.583 \pm 00.016 \\
(9)\end{array}$ & $42.7 \pm 1.3$ & & $\begin{array}{l}-0.7 \pm 00.7 \\
(5)\end{array}$ \\
\hline
\end{tabular}

$\tau$, Labeling rate with Alexa Fluor $488 C_{5}$-maleimide measured as in Fig. 1D; $r_{d}$ and $r_{\text {a }}$ anisotropy for Alexa Fluor $488 C_{5}$-maleimide-labeled and TMR-MTS-labeled transporters, respectively. FRET efficiencies $E$ for the different cysteine residues were estimated by the donor-quenching method (Fig. 1E). Single cysteine: intersubunit distance, $R$, between the same cysteine residue in neighboring subunits estimated from the FRET efficiencies using Equation 1 , and distance to the center, $L$, for the cysteine, assuming a trimeric structure. Cysteine pairs: intrasubunit distances, $R$, between two cysteines in one subunit estimated from the FRET efficiencies using Equation 2 . $\Delta R$ is the change in distance induced by changing the extracellular solution from a choline-Ringer to a Na-Ringer solution with $1 \mathrm{~mm}$ glutamate. $\Delta R$ was measured for each individual oocyte and averaged. That $\Delta R$ was significantly different from 0 was evaluated with a paired $t$ test: ${ }^{*} p<$ 0.05 and ${ }^{* *} p<0.01$. The oocytes were clamped to $-80 \mathrm{mV}$. Values are given as mean \pm SD $(n)$. ND, Not determined.

binding sites. Furthermore, we show that, despite the multimeric nature of glutamate transporters, the subunits function independently.

\section{Materials and Methods}

Expression of EAAT3 transporters

All of the experiments were done on the human glutamate transporter EAAT3. Site-directed mutagenesis, in vitro synthesis of RNA, and RNA injection into Xenopus laevis oocytes were performed as described previously (Larsson et al., 2004). For the intrasubunit FRET measurements, we inserted two cysteines in one subunit and injected wild-type (wt) to double-mutant RNA at a ratio of 10:1. This ratio ensured that a majority of the fluorescent-labeled transporters had only one fluorescent-labeled subunit and that the intrasubunit FRET measurements were only minimally contaminated by intersubunit FRET. For a trimeric protein, the probability, $\mathrm{p}(\mathrm{n})$, of having $n$ subunits with the introduced cysteines are as follows: $\mathrm{p}(3)=0.1^{3}=0.1 \% ; \mathrm{p}(2)=3 \times 0.1^{2} \times 0.9=2.7 \% ; \mathrm{p}(1)=3 \times$ $0.1 \times 0.9^{2}=24.3 \%$; and $\mathrm{p}(0)=0.9^{3}=72.9 \%$. All of the cysteineintroduced transporters expressed equal or less than the wt EAAT3, as judged by the size of the glutamate-activated currents. Assuming that the protein expressions are proportional to the currents, this means that the ratio of wt/mutant subunits was at least 10:1

\section{Electrophysiology}

Currents were measured with the two-electrode voltage-clamp technique, as described previously (Larsson et al., 2004). Glutamate-activated currents were estimated by subtracting currents measured in a sodium Ringer's solution (in mM: $98.5 \mathrm{NaCl}, 5 \mathrm{HEPES}, 1.8 \mathrm{CaCl}_{2}$, and $1 \mathrm{MgCl}_{2}$, $\mathrm{pH}$ 7.5) from currents measured in a sodium Ringer's solution with $1 \mathrm{mM}$ glutamate.

\section{Labeling of oocytes and fluorescence methods}

Intersubunit FRET measurements. We first measured the labeling kinetics for each residue (see Fig. $1 D$, Table 1). The labeling kinetics varied substantially among the different residues. For the FRET experiments, the oocytes were labeled to $10 \%$ of maximum fluorescence labeling with Alexa Fluor $488 \mathrm{C}_{5}$-maleimide (Molecular Probes, Eugene, OR) in Ringer's solution (see Fig. $1 D)$. This ensured that only $10 \%[0.027 /(0.027+$ $0.243)]$ of the subunits that were labeled with donor fluorophores had two donors, because the probability, $\mathrm{p}(\mathrm{n})$, of having $n$ donor fluorophores are as follows for a trimeric protein: $\mathrm{p}(3)=0.1^{3}=0.1 \% ; \mathrm{p}(2)=$ $3 \times 0.1^{2} \times 0.9=2.7 \% ; \mathrm{p}(1)=3 \times 0.1 \times 0.9^{2}=24.3 \%$; and $\mathrm{p}(0)=$ $0.9^{3}=72.9 \%$. The transporters with two donor fluorophores also underwent FRET but to a slightly lesser extent than the transporters with only one donor fluorophore. This introduced a maximum error of $2.5 \%$ in the 
distance estimate between residues from the FRET efficiencies (supplemental material, available at www.jneurosci.org).

Intrasubunit FRET measurements. We used pairs of residues with large differences in labeling kinetics, which enabled us to specifically label one residue without significantly labeling the second residue ( $<10 \%$ double labeling). The cysteine with the fastest labeling kinetics of the two cysteines was labeled to $50 \%$ of maximum fluorescence labeling with Alexa Fluor $488 \mathrm{C}_{5}$-maleimide in Ringer's solution. Subunits labeled with two donor fluorophores did not undergo FRET. Therefore, in estimating the FRET efficiencies, the donor fluorescence was corrected for this predicted double labeling of donor fluorophores.

In both types of FRET measurements, the donor-labeled oocyte was placed on a Zeiss (Oberkochen, Germany) LSM 510 inverted confocal microscope, and the fluorescence spectrum was measured using the Zeiss META detector exciting with a $488 \mathrm{~nm}$ argon laser. The oocyte was then labeled to saturation with tetramethylrhodamine-methanethiosulfonate (TMR-MTS) (Toronto Research Chemicals, Toronto, Ontario, Canada), and the fluorescence spectrum was measured again after the nonbound rhodamine was washed out.

Spectra were measured in a choline solution (in mu: 98.5 cholineCl, 5 HEPES, $1.8 \mathrm{CaCl}_{2}$, and $1 \mathrm{MgCl}_{2}, \mathrm{pH} 7.5$ ), a sodium Ringer's solution, and a sodium Ringer's solution with $1 \mathrm{~mm}$ glutamate before and after the rhodamine application. FRET efficiencies were measured as the decrease of the donor (Alexa Fluor 488) fluorescence caused by the acceptors (rhodamine) (Selvin, 1995). The decrease of donor fluorescence was measured at $520 \mathrm{~nm}$ because, at this wavelength, the oocyte endogenous fluorescence and the acceptor fluorescence were negligible when excited with a $488 \mathrm{~nm}$ laser (see Fig. $1 D$ ). To estimate distances in the intersubunit FRET measurements (one donor and two acceptor fluorophores), the following equation was used: $E=k_{\mathrm{FRET}} /\left(1 / \tau_{\mathrm{D}}+k_{\mathrm{FRET}}\right)$, where $k_{\mathrm{FRET}}=2 / \tau_{\mathrm{D}} \times\left(R_{0} / R\right)^{6}, k_{\mathrm{FRET}}$ is the energy transfer rate, and $R_{0}$ (the Förster distance) is given by $R_{0}=0.211\left(\kappa^{2} n^{-4} Q_{\mathrm{D}} J(\lambda)\right)^{-1 / 6}$, where $\kappa$ is the orientation factor, $n$ is the refractive index of the solvent, $Q$ is the quantum yield, and $J(\lambda)$ is the spectral overlap between the donor emission and acceptor absorption. $R_{0}$ was estimated for Alexa Fluor 488 and TMR to be 50 $\AA$, and $\kappa^{2}$ was assumed to be $2 / 3$. Uncertainties in the $\kappa^{2}$ lead to errors in the estimates of $R_{0}$. We therefore measured the anisotropy (Table 1 ) to estimate the range of possible values of $\kappa^{2}$ for all of the residues. $\kappa^{2}$ was assumed to be $2 / 3$, because the anisotropy measurements indicated that all of the residues had a reasonable mobility. The range of possible values of $\kappa^{2}$ was $0.5-1.2$ for the residue with the lowest anisotropy and 0.3-2 for the residue with the highest anisotropy. These ranges correspond to a worst-case error in $R_{0}$ of 5 and $10 \AA$, respectively. Most likely, the errors are smaller because the estimates of $\kappa^{2}$ do not take into account the depolarization attributable to the FRET transfer process itself (Lakowicz, 1999). We have shown previously that the fluorescence from fluorophores attached to A430C in EAAT3 (in the absence of an acceptor fluorophore) changes in response to conformational changes (Larsson et al., 2004). For all of the different Alexa Fluor 488-labeled residues, the changes in fluorescence intensities were less than a few percent (data not shown). Assuming that the quantum yield, $Q_{\mathrm{D}}$, is proportional to the fluorescence intensities, the quantum yield changes only by a few percent. Because $R_{0}$ is proportional to $\left(Q_{D}\right)^{1 / 6}$, even a $10 \%$ change in $Q_{D}$ would alter $R_{0}$ by $<1 \AA$. We have therefore not included any correction factor of $R_{0}$ as a result of changes in $Q_{\mathrm{D}}$ in our analysis.

To estimate distances in the intrasubunit FRET measurements (one donor and one acceptor fluorophore), the following equation was used: $E=k_{\mathrm{FRET}} /\left(1 / \tau_{\mathrm{D}}+k_{\mathrm{FRET}}\right)$, where $k_{\mathrm{FRET}}=1 / \tau_{\mathrm{D}} \times\left(R_{0} / R\right)^{6}$.

\section{Anisotropy and $\kappa^{2}$ range}

The anisotropy $(r)$ was measured from oocytes labeled with only Alexa Fluor $488 \mathrm{C}_{5}$-maleimide $\left(r_{\mathrm{d}}\right)$ and oocytes labeled with only TMR-MTS $\left(r_{\mathrm{a}}\right) \cdot r=\left(I_{\|}-I_{\perp}\right) /\left(I_{\|}+2 I_{\perp}\right)$, where $I_{\|}$is the parallel and $I_{\perp}$ is the perpendicular-emitted light with respect to the polarized excitation light. The collimated emission signal was split into parallel and perpendicular components by using a polarizing cube beam splitter, and the two components were measured simultaneously. The error bound in the distance measurements was calculated by setting upper and lower limits for $\kappa^{2}$ by using anisotropy values for the donor $\left(r_{\mathrm{d}}\right)$ and acceptor $\left(r_{\mathrm{a}}\right): \kappa^{2}$ max $=$ $2 / 3\left(1+F r_{\mathrm{d}}+F r_{\mathrm{a}}+3 F r_{\mathrm{d}} \times F r_{\mathrm{a}}\right)$ and $\kappa^{2}{ }_{\min }=2 / 3\left(1-\left(F r_{\mathrm{d}}+F r_{\mathrm{a}}\right) / 2\right)$, where $F r_{\mathrm{d}}$ and $F r_{\mathrm{a}}$ are defined as $\left(r_{\mathrm{d}} / r_{0}\right)^{0.5}$ and $\left(r_{\mathrm{a}} / r_{0}\right)^{0.5}$, respectively. $r_{0}$ is the fundamental anisotropy (Lakowicz, 1999).

\section{Modeling}

The distance estimates between residues from the FRET efficiencies were interpreted in a two-dimensional model by assuming that all of the residues are located in an $x-y$ plane parallel to the cell membrane in a symmetrical structure. If residues are located at different distances from the membrane $(z)$, then our FRET measurements will overestimate the intrasubunit distances in the $x-y$ plane, and thus the residues will seem farther apart in the subunit in our model versus in a three-dimensional model. This assumption would affect only the intrasubunit distance estimates because the intersubunit distance estimates are between like residues in different subunits.

\section{Results}

Hydropathy plots and cysteine-accessibility studies suggest that the $\mathrm{C}$ and $\mathrm{N}$ termini of glutamate transporters are located in the cytosol and that the transporters have eight $\alpha$-helical transmembrane domains and one or two membrane-inserted loops (Grunewald et al., 1998; Seal and Amara, 1998; Grunewald and Kanner, 2000; Seal et al., 2000) (Fig. 1A). Studies using chemical cross-linking reagents showed that glutamate transporters form multimeric structures, most likely trimers (Haugeto et al., 1996; Dehnes et al., 1998). Recent biochemical studies also suggested that the bacterial glutamate transporter and human EAAT2 are trimers (Haugeto et al., 1996; Yernool et al., 2003; Gendreau et al., 2004). However, freeze-fracture electron microscopy images suggest that the neuronal transporter EAAT3 assembles into a pentamer (Eskandari et al., 2000).

To determine the relative positions of the different transmembrane domains and membrane-inserted loops in the human neuronal glutamate transporter EAAT3, we used FRET analysis on EAAT3 transporters expressed in Xenopus oocytes. We introduced the fluorophores by inserting cysteine residues in different 


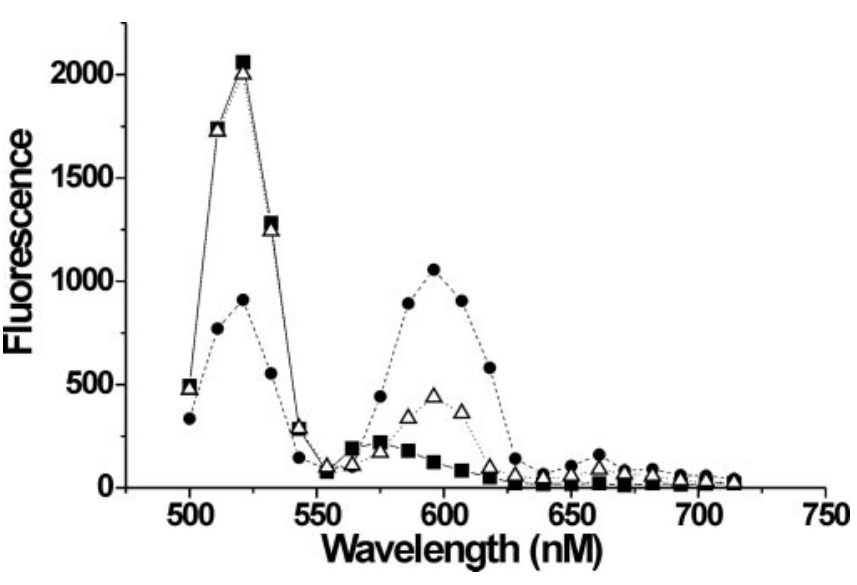

Figure 2. The quenched donor fluorescence is restored by DTT. Emission spectra for an 00cyte expressing EAAT3 M60C transporters: unquenched Alexa Fluor 488 before application of TMR-MTS ( $\mathbf{\square}$, solid line), quenched Alexa Fluor 488 after application of TMR-MTS ( , dashed line), and dequenched Alexa Fluor 488 after a subsequent application of $10 \mathrm{~mm}$ DTT for $10 \mathrm{~min}$ ( $\triangle$, dotted line). DTT reduces the disulfide between cysteines and TMR-MTS but cannot remove the Alexa Fluor $488 C_{5}$-maleimides. DTT restores the donor fluorescence to its original value.

parts of EAAT3 (Fig. 1A) and then labeled the cysteines with cysteine-reactive fluorescent probes. All 19 of the cysteine-substituted EAAT3 gave similar glutamate-activated uptake currents as wildtype EAAT3 (Fig. $1 B$ ), suggesting that the cysteine substitutions did not significantly alter the function of EAAT3. We estimated the FRET efficiency by the donor-quenching method (Selvin, 1995), using Alexa Fluor 488 as the donor and tetramethylrhodamine as the acceptor. In the donor-quenching method, the percentage decrease in donor fluorescence caused by the presence of the acceptor fluorophore is a direct estimate of the FRET efficiency. We started by measuring intersubunit distances between cysteines introduced at the same residue in all of the subunits.

Our strategy was to label only one subunit with a donor fluorophore in each transporter multimer and then to label the remaining subunits with acceptor fluorophores (Fig. $1 C$ ). We wanted a homogenous population of donor-labeled EAAT3 to simplify the distance estimates in the FRET analysis. To ensure that we labeled only one subunit per transporter with the donor fluorophore, we first measured the labeling kinetics for each cysteine mutation (Fig. 1D, Table 1). From the labeling curve, we calculated the exposure to the donor fluorophore needed to label, on average, only $10 \%$ of the cysteines, which ensured that a majority $(90 \%)$ of the donor-labeled EAAT3 had only one subunit with a donor fluorophore (see Materials and Methods). We then measured the fluorescence from the donor-labeled cells (Fig. 1 E). We then labeled the same cell with a saturating dose of acceptor fluorophores and measured the decrease in donor fluorescence caused by the acceptor fluorophores (Fig. $1 E$ ). The decrease in donor fluorescence was not attributable to bleaching because removing the acceptor fluorophores with $10 \mathrm{~mm}$ DTT (which reduces the bonds between the MTS-linked tetramethylrhodamine fluorophores and cysteines but not the bonds between maleimide-linked Alexa Fluor 488 fluorophores and cysteines) restored the donor fluorescence to its original value (Fig. 2). In addition, subsequent scans with the $488 \mathrm{~nm}$ laser did not have a noticeable bleaching effect on the donor fluorophore Alexa Fluor 488. The percentage of decrease in donor fluorescence gives a direct estimate of the FRET efficiency, thereby giving an estimate of the distance between fluorophores (Selvin, 1995). The distance $(R)$ between fluorophores estimated from the FRET efficiency is not significantly dependent on the number of subunits in the transporter, as long as there are more than two subunits in a transporter (Fig. 1C). This is attributable to the steep distance dependence of the FRET efficiency, which makes the nearestneighbor fluorophores dominate the FRET efficiency.

Table 1 shows the intersubunit FRET efficiencies and the intersubunit distance estimates for a number of cysteines introduced in the extracellular regions of EAAT3. These distance estimates were made under the assumption of a symmetrical structure. The two predicted membrane-inserted loops and TM2 were found to have the highest FRET efficiencies, suggesting that they are closest to the center of the transporter. In contrast, TM1 and TM3 had the smallest FRET efficiencies, suggesting that they are at the perimeter of the transporter. Previous electron microscopy studies have shown that the diameter of EAAT3 is $\sim 76 \AA$ (Eskandari et al., 2000). These previous electron microscopy pictures also suggested that EAAT3 is a pentameric structure (Eskandari et al., 2000), whereas biochemical cross-linking studies of bacterial and human EAAT2 glutamate transporters suggested a trimeric structure (Haugeto et al., 1996; Yernool et al., 2003; Gendreau et al., 2004). We found all of the intersubunit distances to be in the range of $49-75 \AA$. This finding is most consistent for a trimeric structure of EAAT3. For a pentameric structure with a diameter of $\sim 80 \AA$, no intersubunit distances would be $>45 \AA$ (Eskandari et al., 2000).

Curiously, we did not find any residue with an intersubunit distance of $<49 \AA$, suggesting that the extracellular part of EAAT3 that we tested is doughnut shaped. One possible explanation for the lack of residues with short intersubunit distances is that there is a large (width, $>45 \AA$ ) central vestibule at the extracellular surface of EAAT3. Other possible explanations are that the central portion of EAAT3 is filled with lipid or that some parts of the large loop between TM3 and TM4, which we have not tested, form the center of the glutamate transporter.

We then measured intrasubunit FRET between cysteine pairs introduced in the same subunit. The subunits with two cysteine mutations were coexpressed with an excess of wild-type subunits (1:10 ratio), to minimize contamination by intersubunit FRET (see Materials and Methods). Table 1 shows the intrasubunit FRET efficiencies and the intrasubunit distance estimates for a number of cysteine pairs that were introduced in a single subunit of EAAT3. We also conducted control experiments in which subunits with only one cysteine mutation were coexpressed with an excess of wild-type subunits (1:10 ratio) and were labeled with an identical protocol as that for the intrasubunit FRET experiments. This coexpression did not show any significant FRET $(2 \% ; n=$ 5 ), in contrast to the coexpression of the double-cysteine subunits with wild-type subunits (intrasubunit FRET) (Table 1). This suggests that there is no significant contamination of intersubunit FRET (nor intertransporter FRET) in our intrasubunit FRET measurements. Figure $3 A$ shows our model, which takes into account both the intersubunit and the intrasubunit distance estimates. Since the submission of this paper, a crystal structure of a putative glutamate transporter from bacteria has been published (Yernool et al., 2004). In Figure 3, we compare our distance estimates with the distances measured in the crystal structure for homologous residues. Our distance estimates and the distances in the crystal structure correlate well (Fig. $3 C$ ), suggesting that the overall structure is conserved between the bacterial and the human glutamate transporters (Fig. 3).

We then tested whether EAAT3 undergoes large-scale molecular motions during glutamate uptake, such as the "rockerswitch" motion proposed for the LacY (Abramson et al., 2003) and 

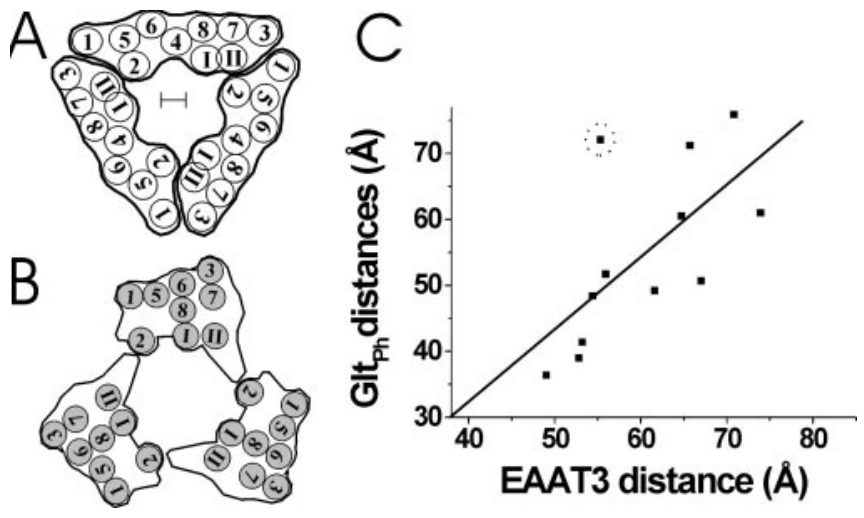

Figure 3. Conservation of glutamate transporter structure between bacteria and mammals. $A$, Extracellular view of a trimeric EAAT3 model that is mostly consistent with distances estimated from our FRET measurements (Table 1). Uncertainties exist in the location of the residues relative to the $x-y$ plane, making a more detailed model impossible (see Materials and Methods). Scale bar, $10 \AA$. B, Extracellular view of the putative bacterial transporter $\mathrm{Glt}_{\mathrm{PH}}$ (Yernool et al., 2004). The extracellular positions of the different transmembrane domains are indicated by the circles. C, A strong correlation exists between the intersubunit distances estimated with FRET (EAAT3 distances) and the distances in the crystal structure (Glt $t_{\mathrm{Ph}}$ distances) measured between the homologous residues (measured at the $\alpha$-C atoms) in neighboring subunits. The FRET estimates are, on average, $8 \AA$ longer, most likely attributable to the size of the linker and the fluorescent probes. The distance between $L 387$ C residues (circled) deviates considerably from the distance between the homologous residues in the crystal structure. Note that this residue undergoes the largest movement according to our FRET measurements (Table 1) and is directly connected to loop II (Fig. 1A), which has been shown to undergo several conformational changes during the uptake cycle (Larsson et al., 2004).

the glycerol-3-phosphate secondary transporters (GlpT) (Huang et al., 2003), or large, rotational motions (up to $50 \AA$ ) suggested in P-type ATPases (Kuhlbrandt et al., 2002; Toyoshima and Nomura, 2002; Toyoshima and Inesi, 2004; Toyoshima and Mizutani, 2004). We measured FRET efficiency under conditions that should favor extracellular-facing (100 mM extracellular choline ${ }^{+}$) and intracellular-facing glutamate-binding sites ( $100 \mathrm{~mm}$ extracellular $\mathrm{Na}^{+}$and 1 mM extracellular glutamate). In the most recent kinetic model of EAAT3 (Larsson et al., 2004), in $100 \mathrm{~mm}$ choline ${ }^{+}$, the transporter spends $100 \%$ of its time with the binding sites facing the extracellular solution. In contrast, in $100 \mathrm{~mm}$ extracellular $\mathrm{Na}^{+}$and $1 \mathrm{~mm}$ extracellular glutamate, the transporter spends $>90 \%$ of its time with the binding sites facing the intracellular solution.

The application of glutamate induced currents in all of the labeled mutations (except S334C). The application of glutamate either did not significantly change or produced only small changes in the FRET efficiencies in the intersubunit or intrasubunit experiments (Table 1). These findings suggest that glutamate uptake is not accomplished by large-scale motions, such as those motions proposed from the crystal structures of the LacY and GlpT transporters (Abramson et al., 2003; Huang et al., 2003). Rather, glutamate uptake may be accomplished by relatively small-scale motions in each subunit. Using voltage-clamp fluorometry, we have shown previously that A430C in loop II, a region that has been hypothesized to be part of the substrate-binding site (Slotboom et al., 1999; Kanner and Borre, 2002), undergoes several conformational changes during the glutamate-uptake cycle (Larsson et al., 2004). However, our FRET experiments indicate that these conformational changes are relatively small $(<5 \AA)$.

Our results also indicated that the residues in loop II, TM7, and TM8 that have been hypothesized to be part of the glutamatebinding site (Slotboom et al., 1999; Kanner and Borre, 2002) are located far apart ( $>45 \AA$ ) from the same residues in a neighboring subunit. This finding suggests that there is one glutamate-

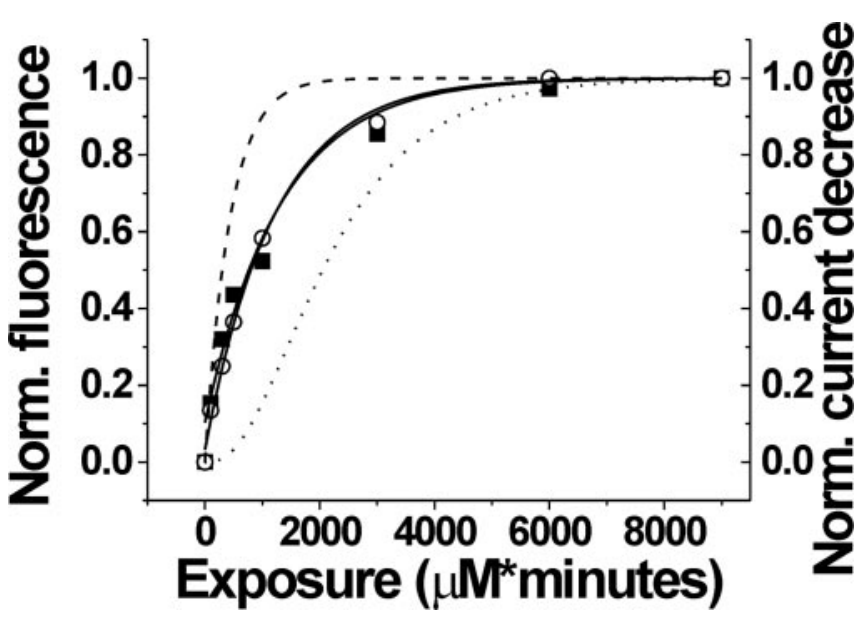

Figure 4. EAAT3 subunits function independently. Labeling curve for 334C with Alexa Fluor 488 maleimide $(\square)$ superimposed on the percentage of decrease in glutamate-activated current caused by the fluorescent label $(\bigcirc)$, shown for a representative oocyte $(n=3)$. Solid lines are the best exponential fit to the data: $\tau_{\text {fluorescence }}=1306 \pm 182 \mu \mathrm{M} \times \min ; \tau_{\text {current decrease }}=1208 \pm 62$ $\mu \mathrm{M} \times \mathrm{min}$. The dashed line is the predicted decrease in current for a concerted cooperative trimeric transporter. The dotted line indicates the predicted decrease in current for a trimeric transporter in which only one glutamate-binding site can be occupied at a time, and any one of the binding sites can induce the glutamate-activated current through a common pathway. Norm., Normal.

binding site per subunit and that each transporter multimer can bind several glutamate molecules (three glutamates for one trimeric structure). However, it is unknown whether the subunits function independently or cooperatively (DeFelice, 2004). Our data, showing that the putative glutamate-binding sites are far apart and that uptake is accomplished without large-scale (intersubunit) conformational changes suggest that the binding sites function independently. The glutamate-activated currents from one of our cysteine mutations (S334C) were abolished when the cysteine was labeled with a fluorescent probe, which allowed us to use S334C labeling to test whether the subunits function independently. This cysteine mutation had a high FRET efficiency (Table 1), showing that we can label more than one subunit in an S334C transporter multimeric structure. If the transporter subunits function independently, then we predict that the reduction of the glutamate-activated current caused by fluorescent labeling and the increase in total fluorescent labeling will correlate 1:1 for all of the labeling conditions. In contrast, if the transporter subunits function cooperatively, then we predict that the reduction of the glutamate-activated current caused by fluorescent labeling will be greater than the increase in total fluorescent labeling for the shorter labeling times. Figure 4 shows that the reduction of the glutamate-activated current caused by fluorescent labeling and the increase in total fluorescent label correlated 1:1 for all of our labeling conditions, indicating that the EAAT3 subunits function independently.

\section{Discussion}

In our FRET experiments, we found that all of the intersubunit distances were in the range of 49-75 $\AA$. This finding is the most consistent for a trimeric structure of EAAT3 because, for a pentameric structure with a diameter of $\sim 80 \AA$, no intersubunit distances would be $>45 \AA$ (Eskandari et al., 2000). Since the submission of this paper, a crystal structure of a putative glutamate transporter $\left(\mathrm{Glt}_{\mathrm{Ph}}\right)$ from bacteria has been published (Yernool et al., 2004). Our distance estimates and the distances in the crystal structure correlate well (Fig. 3C), suggesting that the overall structure is conserved between the bacterial and the human 

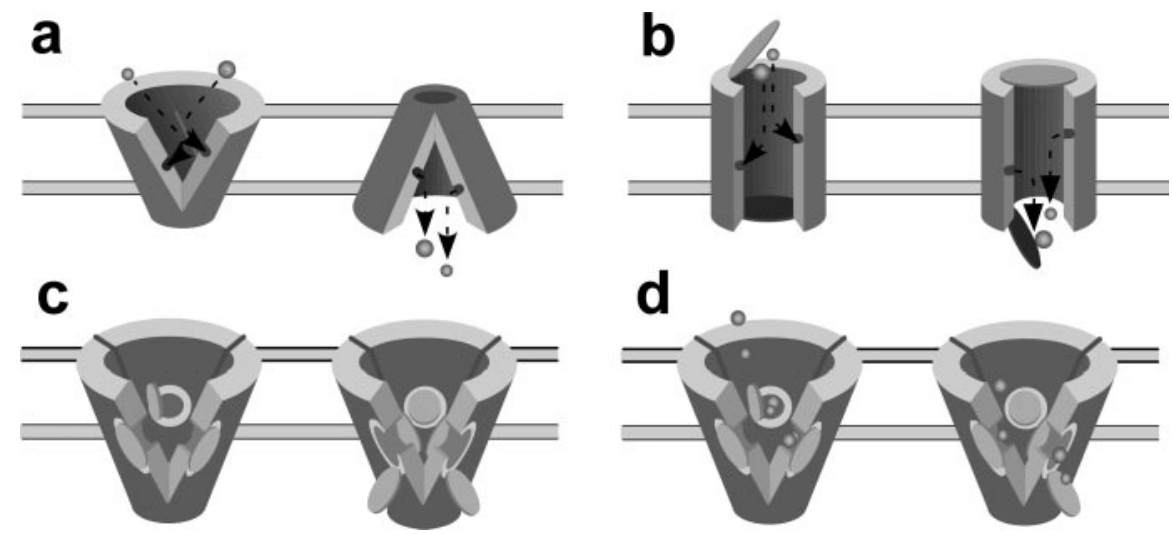

Figure 5. Molecular models of glutamate transporters. $a$, A rocker-switch-type model for transport, which alternately exposes the glutamate-binding site to the intracellular and extracellular solutions by rotating the subunits relative each other. $b, A$ channel model for transport with two gates that open alternately, allowing coupled transport. c, EAAT3 model with a large, extracellular vestibule and three short channels, each with two gates. The gates alternately open to the extracellular solution (left) or to the intracellular solution (right). $d$, Our data indicate that the three pores transport glutamate independently. Large sphere, glutamate; small sphere, $\mathrm{Na}^{+}$. For simplicity, only one $\mathrm{Na}^{+}$and one glutamate are shown to be transported for each transport cycle. It was shown previously that, in each cycle, three $\mathrm{Na}^{+}$, one $\mathrm{H}^{+}$, and one glutamate are cotransported, whereas one $\mathrm{K}^{+}$is countertransported (Zerangue and Kavanaugh, 1996; Levy et al., 1998).

glutamate transporters (Fig. 3). The bacterial $\mathrm{Glt}_{\mathrm{Ph}}$ transporter has a large extracellular vestibule (Yernool et al., 2004). We did not find any residue with an intersubunit distance of $<49 \AA$, suggesting that there is also a large (width, $>45 \AA$ ) central vestibule at the extracellular surface of EAAT3. Another possibility is that some parts of the large loop between TM3 and TM4, which we have not tested, form the center of the glutamate transporter. This loop is much shorter in the bacterial transporter, but the central location of the TM4 in the crystal structure suggests that this loop might fill part of the external vestibule (Yernool et al., 2004). The distance between L387C residues deviates considerably from the distance between the homologous residues in the crystal structure of the $\mathrm{Glt}_{\mathrm{Ph}}$ transporter. However, this residue undergoes the largest movement according to our FRET measurements (Table 1) and is directly connected to loop II (Fig. 1A), which has been shown to undergo several conformational changes during the uptake cycle (Larsson et al., 2004). One possibility for the discrepancy between the FRET and the crystal structure distance for L387 is that the protein in the crystal structure is in a short-lived or rare conformational state, whereas the FRET measurements are intrinsically biased to report the more long-lived conformational states. Another possibility is that the structures of the human and the bacterial transporters are different in this region.

Transport of one glutamate molecule is coupled to the cotransport of three sodium ions and one proton and to the countertransport of one potassium ion (Zerangue and Kavanaugh, 1996; Levy et al., 1998). The exact molecular mechanism for this coupled transport of substrates is not well understood. It is thought that glutamate transporters mediate glutamate transport via a reaction cycle with conformational changes between the two major access states that alternately expose glutamate-binding sites to the extracellular or to the intracellular solution. Two types of models have been suggested for the alternating-access transport mechanism in secondary active-transporter proteins (for review, see DeFelice, 2004): the rocker-switch model (Fig. 5A) (Jardetzky, 1966; Abramson et al., 2003; Huang et al., 2003) and the "two-gated channel" model (Fig. 5B) (Vidaver, 1966; Lauger, 1979; Lester et al., 1994, 1996; Cao et al., 1998). The rocker-switch model, in which whole domains rotate relative to each other to alternate the exposure of the substratebinding sites located in large extracellular and intracellular vestibules, predicts that large-scale motions underlie the substrateuptake mechanism. In contrast, the twogated channel model, in which local smallscale motions open and close two gates at the opposite ends of a diffusion channel for the substrates, predicts that small-scale motions underlie the substrate-uptake mechanism.

Several groups have shown that glutamate transporters undergo conformational changes. Grunewald and Kanner (1995) showed that transported substrates induce conformational changes that expose additional trypsin sites in the glial transporter GLT-1/EAAT2. Several groups have measured the accessibility of cysteinespecific reagents to cysteines introduced at different sites in glutamate transporters, in the presence and absence of substrates (Slotboom et al., 1996, 1999, 2001; Grunewald et al., 1998; Seal et al., 1998, 2000; Zarbiv et al., 1998; Grunewald and Kanner, 2000). They have shown that a number of residues display substrate-dependent accessibility. Using voltage-clamp fluorometry, we have shown previously that $\mathrm{A} 430 \mathrm{C}$ in loop II, a region that has been hypothesized to be part of the substrate-binding site (Slotboom et al., 1999; Kanner and Borre, 2002), undergoes several conformational changes during the glutamate-uptake cycle (Larsson et al., 2004). Although these studies showed that glutamate transporters undergo conformational changes, the limited structural information in these studies made it difficult to draw specific conclusions about the nature of the conformational changes in glutamate transporters.

The application of glutamate either did not significantly change or produced only small changes in the FRET efficiencies in the intersubunit or intrasubunit experiments (Table 1). These findings suggest that glutamate uptake is not accomplished by large-scale motions.

We therefore suggest that EAAT3 glutamate transporters have a large, extracellular vestibule, as in the extracellular-facing state in the rocker-switch-type model, but that glutamate transporters do not undergo a large-scale motion to the other putative (intracellularfacing) state in the rocker-switch-type model, as suggested for other transporter proteins (Abramson et al., 2003; Huang et al., 2003). In contrast, our data suggest that small-scale motions accomplish the glutamate uptake. Therefore, we suggest a hybrid model in which short channels connect the intracellular solution to the large extracellular vestibule (Fig. 5C). This model would allow small conformational changes to alternately expose the same glutamate-binding sites to the intracellular and the extracellular solutions, without the need for glutamate to diffuse large distances in the protein (Fig. 5D). Our results indicate further that these conformational changes, and hence glutamate uptake, occur independently in each subunit (Fig. $5 D$ ). The reason for the multimeric assembly of glutamate transporters is not clear from our data. One possibility is that the trimeric structure allows for the formation of the large external vestibule that facilitates the diffusion of charged substrates (such as $\mathrm{Na}^{+}$and glutamate $^{-}$) deep into the membrane (Yernool et al., 2004). This structural feature and the small-scale conformational changes suggested by our data could contribute to the fast kinetics predicted for glutamate transporters (Otis and Jahr, 1998). 


\section{References}

Abramson J, Smirnova I, Kasho V, Verner G, Kaback HR, Iwata S (2003) Structure and mechanism of the lactose permease of Escherichia coli. Science 301:610-615.

Arriza JL, Fairman WA, Wadiche JI, Murdoch GH, Kavanaugh MP, Amara SG (1994) Functional comparisons of three glutamate transporter subtypes cloned from human motor cortex. J Neurosci 14:5559-5569.

Arriza JL, Eliasof S, Kavanaugh MP, Amara SG (1997) Excitatory amino acid transporter 5 , a retinal glutamate transporter coupled to a chloride conductance. Proc Natl Acad Sci USA 94:4155-4160.

Bergles DE, Diamond JS, Jahr CE (1999) Clearance of glutamate inside the synapse and beyond. Curr Opin Neurobiol 9:293-298.

Cao Y, Li M, Mager S, Lester HA (1998) Amino acid residues that control $\mathrm{pH}$ modulation of transport-associated current in mammalian serotonin transporters. J Neurosci 18:7739-7749.

Chaudhry FA, Lehre KP, van Lookeren Campagne M, Ottersen OP, Danbolt NC, Storm-Mathisen J (1995) Glutamate transporters in glial plasma membranes: highly differentiated localizations revealed by quantitative ultrastructural immunocytochemistry. Neuron 15:711-720.

Danbolt NC (2001) Glutamate uptake. Prog Neurobiol 65:1-105.

DeFelice LJ (2004) Transporter structure and mechanism. Trends Neurosci 27:352-359.

Dehnes Y, Chaudhry FA, Ullensvang K, Lehre KP, Storm-Mathisen J, Danbolt NC (1998) The glutamate transporter EAAT4 in rat cerebellar Purkinje cells: a glutamate-gated chloride channel concentrated near the synapse in parts of the dendritic membrane facing astroglia. J Neurosci 18:3606-3619.

Eskandari S, Kreman M, Kavanaugh MP, Wright EM, Zampighi GA (2000) Pentameric assembly of a neuronal glutamate transporter. Proc Natl Acad Sci USA 97:8641-8646.

Fairman WA, Vandenberg RJ, Arriza JL, Kavanaugh MP, Amara SG (1995) An excitatory amino-acid transporter with properties of a ligand-gated chloride channel. Nature 375:599-603.

Gendreau S, Voswinkel S, Torres-Salazar D, Lang N, Heidtmann H, DetroDassen S, Schmalzing G, Hidalgo P, Fahlke C (2004) A trimeric quaternary structure is conserved in bacterial and human glutamate transporters. J Biol Chem 279:39505-39512.

Grunewald M, Kanner B (1995) Conformational changes monitored on the glutamate transporter GLT-1 indicate the existence of two neurotransmitter-bound states. J Biol Chem 270:17017-17024.

Grunewald M, Kanner BI (2000) The accessibility of a novel reentrant loop of the glutamate transporter GLT-1 is restricted by its substrate. J Biol Chem 275:9684-9689.

Grunewald M, Bendahan A, Kanner BI (1998) Biotinylation of single cysteine mutants of the glutamate transporter GLT-1 from rat brain reveals its unusual topology. Neuron 21:623-632.

Haugeto O, Ullensvang K, Levy LM, Chaudhry FA, Honore T, Nielsen M, Lehre KP, Danbolt NC (1996) Brain glutamate transporter proteins form homomultimers. J Biol Chem 271:27715-27722.

Huang Y, Lemieux MJ, Song J, Auer M, Wang DN (2003) Structure and mechanism of the glycerol-3-phosphate transporter from Escherichia coli. Science 301:616-620

Jardetzky O (1966) Simple allosteric model for membrane pumps. Nature 211:969-970

Kanai Y, Hediger MA (1992) Primary structure and functional characterization of a high-affinity glutamate transporter. Nature 360:467-471.

Kanai Y, Stelzner M, Nussberger S, Khawaja S, Hebert SC, Smith CP, Hediger MA (1994) The neuronal and epithelial human high affinity glutamate transporter. Insights into structure and mechanism of transport. J Biol Chem 269:20599-20606.

Kanner BI, Borre L (2002) The dual-function glutamate transporters: structure and molecular characterisation of the substrate-binding sites. Biochim Biophys Acta 1555:92-95.

Kuhlbrandt W, Zeelen J, Dietrich J (2002) Structure, mechanism, and regulation of the Neurospora plasma membrane $\mathrm{H}^{+}$-ATPase. Science 297:1692-1696.

Lakowicz JR (1999) Principles of fluorescence spectroscopy, pp 367-394. New York: Kluwer Academic.

Larsson HP, Tzingounis AV, Koch HP, Kavanaugh MP (2004) Fluorometric measurements of conformational changes in glutamate transporters. Proc Natl Acad Sci USA [Erratum (2004) 101:12777] 101:3951-3956.
Lauger P (1979) A channel mechanism for electrogenic ion pumps. Biochim Biophys Acta 552:143-161.

Lester HA, Mager S, Quick MW, Corey JL (1994) Permeation properties of neurotransmitter transporters. Annu Rev Pharmacol Toxicol 34:219-249.

Lester HA, Cao Y, Mager S (1996) Listening to neurotransmitter transporters. Neuron 17:807-810.

Levy LM, Warr O, Attwell D (1998) Stoichiometry of the glial glutamate transporter GLT-1 expressed inducibly in a Chinese hamster ovary cell line selected for low endogenous $\mathrm{Na}^{+}$-dependent glutamate uptake. J Neurosci 18:9620-9628.

Lin CL, Tzingounis AV, Jin L, Furuta A, Kavanaugh MP, Rothstein JD (1998) Molecular cloning and expression of the rat EAAT4 glutamate transporter subtype. Brain Res Mol Brain Res 63:174-179.

Otis TS, Jahr CE (1998) Anion currents and predicted glutamate flux through a neuronal glutamate transporter. J Neurosci 18:7099-7110.

Pines G, Danbolt NC, Bjoras M, Zhang Y, Bendahan A, Eide L, Koepsell H, Storm-Mathisen J, Seeberg E, Kanner BI (1992) Cloning and expression of a rat brain L-glutamate transporter. Nature 360:464-467.

Rothstein JD, Martin L, Levey AI, Dykes-Hoberg M, Jin L, Wu D, Nash N, Kuncl RW (1994) Localization of neuronal and glial glutamate transporters. Neuron 13:713-725.

Seal RP, Amara SG (1998) A reentrant loop domain in the glutamate carrier EAAT1 participates in substrate binding and translocation. Neuron 21:1487-1498.

Seal RP, Daniels GM, Wolfgang WJ, Forte MA, Amara SG (1998) Identification and characterization of a cDNA encoding a neuronal glutamate transporter from Drosophila melanogaster. Receptors Channels 6:51-64.

Seal RP, Leighton BH, Amara SG (2000) A model for the topology of excitatory amino acid transporters determined by the extracellular accessibility of substituted cysteines. Neuron 25:695-706.

Selvin PR (1995) Fluorescence resonance energy transfer. Methods Enzymol 246:300-334.

Slotboom DJ, Lolkema JS, Konings WN (1996) Membrane topology of the C-terminal half of the neuronal, glial, and bacterial glutamate transporter family. J Biol Chem 271:31317-31321.

Slotboom DJ, Sobczak I, Konings WN, Lolkema JS (1999) A conserved serine-rich stretch in the glutamate transporter family forms a substratesensitive reentrant loop. Proc Natl Acad Sci USA 96:14282-14287.

Slotboom DJ, Konings WN, Lolkema JS (2001) Cysteine-scanning mutagenesis reveals a highly amphipathic, pore-lining membrane-spanning helix in the glutamate transporter GltT. J Biol Chem 276:10775-10781.

Storck T, Schulte S, Hofmann K, Stoffel W (1992) Structure, expression, and functional analysis of a $\mathrm{Na}^{+}$-dependent glutamate/aspartate transporter from rat brain. Proc Natl Acad Sci USA 89:10955-10959.

Tanaka K, Watase K, Manabe T, Yamada K, Watanabe M, Takahashi K, Iwama H, Nishikawa T, Ichihara N, Kikuchi T, Okuyama S, Kawashima N, Hori S, Takimoto M, Wada K (1997) Epilepsy and exacerbation of brain injury in mice lacking the glutamate transporter GLT-1. Science 276:1699-1702.

Toyoshima C, Inesi G (2004) Structural basis of ion pumping by $\mathrm{Ca}^{2+}$ ATPase of the sarcoplasmic reticulum. Annu Rev Biochem 73:269-292.

Toyoshima C, Mizutani T (2004) Crystal structure of the calcium pump with a bound ATP analogue. Nature 430:529-535.

Toyoshima C, Nomura H (2002) Structural changes in the calcium pump accompanying the dissociation of calcium. Nature 418:605-611.

Vidaver GA (1966) Inhibition of parallel flux and augmentation of counter flux shown by transport models not involving a mobile carrier. J Theor Biol 10:301-306.

Watanabe T, Morimoto K, Hirao T, Suwaki H, Watase K, Tanaka K (1999) Amygdala-kindled and pentylenetetrazole-induced seizures in glutamate transporter GLAST-deficient mice. Brain Res 845:92-96.

Yernool D, Boudker O, Folta-Stogniew E, Gouaux E (2003) Trimeric subunit stoichiometry of the glutamate transporters from Bacillus caldotenax and Bacillus stearothermophilus. Biochemistry 42:12981-12988.

Yernool D, Boudker O, Jin Y, Gouaux E (2004) Structure of a glutamate transporter homologue from Pyrococcus horikoshii. Nature 431:811-818.

Zarbiv R, Grunewald M, Kavanaugh MP, Kanner BI (1998) Cysteine scanning of the surroundings of an alkali-ion binding site of the glutamate transporter GLT-1 reveals a conformationally sensitive residue. J Biol Chem 273:14231-14237.

Zerangue N, Kavanaugh MP (1996) Flux coupling in a neuronal glutamate transporter. Nature 383:634-637. 